\title{
Direct and Telescopic Mechanochemical Synthesis of Higher-order Organic-Inorganic Hybrid Cocrystals: Tuning Order, Functionality and Size in Cocrystal Design
}

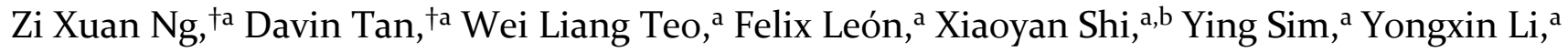 \\ Rakesh Ganguly, ${ }^{\mathrm{a}, \mathrm{c}}$ Yanli Zhao, ${ }^{\mathrm{a}}$ Sharmarke Mohamed, ${ }^{\mathrm{d}, *}$ and Felipe García ${ }^{\mathrm{a}, *}$ \\ a School of Physical and Mathematical Sciences, Division of Chemistry and Biological Chemistry, Nanyang Technological University, 21 Nanyang \\ Link 637371, Singapore (Singapore) \\ b School of Materials and Energy, Guangdong University of Technology, Guangzhou,510oo6, Guangdong, P. R. China. \\ ' Department of Chemistry, Shiv Nadar University, NH91, Tehsil Dadri, Gautam Buddha Nagard, 201314 Uttar Pradesh, India \\ ${ }^{\mathrm{d}}$ Department of Chemistry, Khalifa University of Science and Technology, P.O. Box 127788, Abu Dhabi, UAE
}

\begin{abstract}
The ability to rationally design and predictably construct crystalline solids has been the hallmark of crystal engineering research over the past two decades. When building higher-order multicomponent cocrystals (i.e. crystals containing more than two constituents), the differential and hierarchical way molecules interact and assemble in the solidstate is of pinnacle importance. To date, numerous examples of multicomponent crystals comprising organic molecules leading to salts, cocrystals or ionic cocrystals have been reported. However, the crystal engineering of hybrid organicinorganic cocrystals with sophisticated inorganic building blocks is still poorly understood and mostly unexplored. Here, we reveal the first efficient mechanochemical synthesis of higher-order hybrid organic-inorganic cocrystals based on the structurally versatile - yet largely unexplored - cyclodiphos(V/V)azane heterosynthon building block. The novel hybrid ternary and quaternary multicomponent cocrystals herein reported are held together by synergistic intermolecular interactions (e.g., hydrogen- and halogen-bonding, Se- $\pi$ and ion-dipole interactions). Notably, higher-order ternary and quaternary cocrystals can be readily obtained either via direct synthetic routes from its individual components, or via unprecedented telescopic approaches from lower-order cocrystal sets. In addition, computational modelling has also revealed that the formation of higher-order cocrystals is thermodynamically driven, and that bulk moduli and compressibilities are strongly dependent on the chemical composition and intermolecular forces present in the crystals, which offer untapped potential for optimizing material properties.
\end{abstract}

\section{Introduction}

Cocrystals are multicomponent crystalline solids comprising at least two chemically distinct entities that assemble in a periodic crystalline lattice. ${ }^{[1-4]}$ The rational design and synthesis of multicomponent crystals has been recognized as one of the most challenging aspects of crystal engineering. Whilst supramolecular synthons and energy frameworks have been used to aid the rational discovery of binary cocrystals, the crystal engineering toolkit for the construction of ternary and quaternary cocrystals (and beyond) is not well-established. ${ }^{[5-10]}$ In this context, recent advancements in the development of multicomponent cocrystallization strategies owe largely to their potential as new pharmaceutical solids, as well as functional materials (e.g., optical, luminescent, photomechanical, electronic, inter alia). ${ }^{[1-16]}$ Furthermore, most reported examples of multicomponent crystals relate to the synthesis of organic binary crystals (i.e., defined as salts or cocrystals). ${ }^{[1,2]}$ However, the development of cocrystals based on inorganic building block is still lagging far behind their organic counterparts (vide infra).

Over the past two decades, there has been a fast evolution of the strategies for the design and synthesis of higherorder multicomponent cocrystals. One common strategy is to capitalize on the different intermolecular interactions while taking into consideration the possible hierarchy of the strength and stabilization provided by the intermolecular interaction(s) present (Strategy I). This was demonstrated by Aakeroy et al. where weaker amide...amide hydrogen bonds were preferentially replaced with more thermodynamically favorable heteromeric acid...amide interactions, allowing the formation of ternary cocrystals. ${ }^{[17]}$ This strategy was further discussed by Mandal et al. where they identified the hierarchy of the different intermolecular interactions of hydrogen bonds, halogen bonds, and $\pi-\pi$ stacking through computational simulations. ${ }^{[18]}$ Similarly, Nangia and coworkers used highly robust sulfonamide...pyridineamides...lactam heterosynthons to reproducibly form multicomponent ternary cocrystals. ${ }^{[19]}$

Another strategy for the construction of higher-order cocrystals is the use of isostructurality, and structural inequivalences between supramolecular interactions (Strategy II). Desiraju and co-workers employed supramolecular homologation for the synthesis of organic ternary and quaternary cocrystals, which were subsequently expanded into higher-order molecular solids; their shape-based approach allowed the partial replacement of one component in a binary cocrystal with a structurally similar component to form a ternary crystal, a similar replacement to form a quaternary cocrystals and so on. ${ }^{[20-23]}$ This sequential substitution capitalizes on the varying strengths of the hydrogen bonds, where the more robust interactions persist after the replacement, while the less robust interactions were modified upon the formation of the cocrystal. Similar strategies that utilize size, shape and stoichiometry were employed to create higher-order 
solid solutions. ${ }^{[24-27]}$ In addition, further substitution of lower-order crystal sets can afford five- and sixcomponent molecular solids, hitherto the highest order molecular solids achieved. ${ }^{[21]}$

For more complex molecular systems, Topić and Rissanen $^{[28]}$ utilized a different strategy to construct cocrystals, which involved the combination of synthons comprising robust orthogonal interactions (i.e. interactions that do not interfere with one another) - such as halogen- and hydrogen-bonds (Strategy III). A series of crown ethers, thioureas and perfluorinated halogen bond donors were used as building blocks to obtain various ternary cocrystals that demonstrated perfect orthogonality of the interactions present. The expected stoichiometries and supramolecular motifs were obtained. Their work exemplified the effective design-based strategy to synthesize complex, functional molecular solids from simple building blocks.

On the other hand, despite their lowers number as compared to organic counterparts, inorganic non-carbon frameworks have been shown to be good supramolecular building blocks. Braga and co-workers reported organicinorganic ionic cocrystals (ICCs) as a new class of highly tunable materials ${ }^{[29]}$ that not merely add, but synergistically combine the physicochemical properties of the individual components. ${ }^{[30-31]}$ However, the examples of these type of materials are limited to the use of pure inorganic salts as the inorganic component of the crystal lattice.

Recently, our group has demonstrated the robustness and versatility of cyclodiphosphazanes ${ }^{[32]}$ as a supramolecular building block, ${ }^{[33-34]}$ by synthesizing a series of binary cocrystals, sustained by hydrogen- and halogen-bonding interactions. ${ }^{[34]}$ However their use as inorganic building blocks for the synthesis of "neutral" (i.e., non-ionic) and or ionic multicomponent cocrystaline systems (ICCs) - beyond binary systems - remains unexplored.

With regards to the synthetic methodologies used to synthesize binary cyclodiphosphazane cocrystals, the use of traditional solution-based methodologies continues to dominate. However, there are a number of technical challenges related to the use of solution-based approaches for the synthesis of higher-order cyclodiphosphazane cocrystals. Firstly, the inherent differences in the solubilities of chemical species, limits the choice of solvents that can be employed to successfully obtain higher-order cocrystals. Secondly, to avoid having unsolicited solvent molecules within the crystal lattice, ${ }^{[3]}$ the choices of solvents are further limited. Our previous study has highlighted this challenge, as it demonstrated the high affinity of cyclodiphosphazane for certain solvent molecules, crystallizing readily with a specific solvate molecule despite being in a physical mixture with other components. ${ }^{[34]}$ Furthermore, the use of solution-based methodologies causes a higher probability of separate crystallization of the individual components when attempting to synthesize multicomponent cocrystals. In

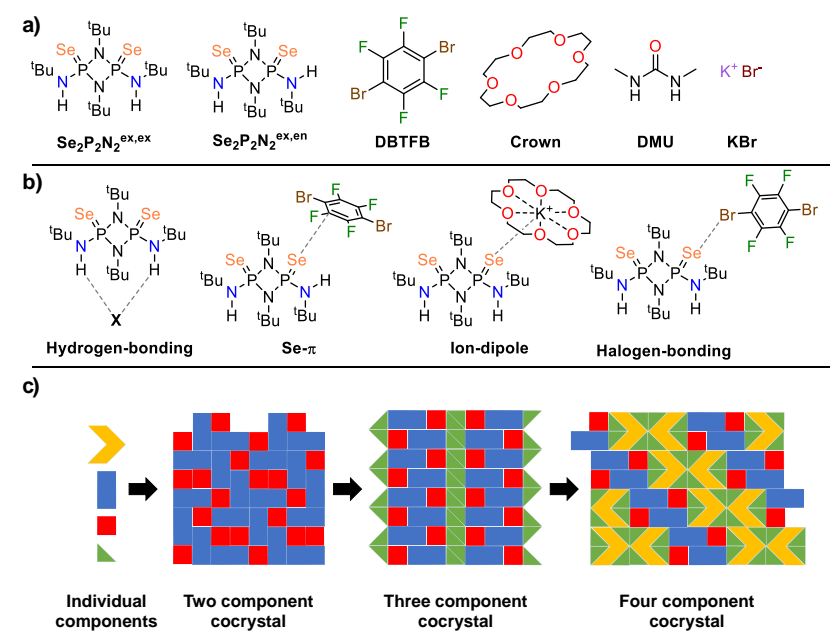

Scheme 1. (a) Molecular structures involved in the synthesis of cocrystals. (b) Supramolecular motifs and interactions displayed by the $\mathrm{Se}_{2} \mathrm{P}_{2} \mathrm{~N}_{2}$ molecule in the presence of various coformers. (c) Schematic illustration of the telescopic mechanosynthesis approach used for the construction of higher-order cocrystals.

contrast to solution-based methods, solid-state mechanochemical grinding has been successfully employed for (i) the rational and targeted synthesis of a range of supramolecular architectures and frameworks, ${ }^{[35-}$ 39] (ii) rapid cocrystal screening and formation, ${ }^{[40-44]}$ and (iii) reproducibly enabling intricate multicomponent cocrystals. ${ }^{[45-49]}$

Building on these precedents - (i) the different cocrystal synthetic strategies (Strategies I-III) previously reported, (ii) the lack of examples high-order cocrystals involving complex inorganic building blocks, and (iii) the proven ability of mechanochemistry in cocrystal synthesis - we set out to rationally crystal engineer of novel hybrid higherorder organic-inorganic cocrystals comprising "complex" cyclophosphazane building blocks by exploiting both the hierarchy of intermolecular interactions, and the use of synthon orthogonality as design principles for their construction (Scheme 1).

Herein, we report the first mechanochemical synthesis of unique ternary and quaternary hybrid organic-inorganic cocrystals. The supramolecular architectures are sustained by either neutral or charge-assisted intermolecular interactions (i.e. hydrogen-bonds, halogen-bonds, iondipole and Se- $\pi$ interactions). In contrast to previous hybrid inorganic-organic cocrystals, our species are based on complex, but easily tunable, inorganic cyclodiphosphazane supramolecular synthons and not just pure inorganic salts.

Certain multi-step chemical transformations reactions can be conducted serially within a single reaction vessel without the need to isolate intermediates. Such a synthetic strategy is coined as a "reaction telescopy" or "telescopic reactions" and is often used by process chemists in organic reactions to form targeted molecules. ${ }^{\left[5^{0}\right]}$ We demonstrate for the first time that targeted multicomponent cocrystals can be achieved in essentially quantitative yield via a onepot telescopic mechanochemical strategy (see Scheme 2). 


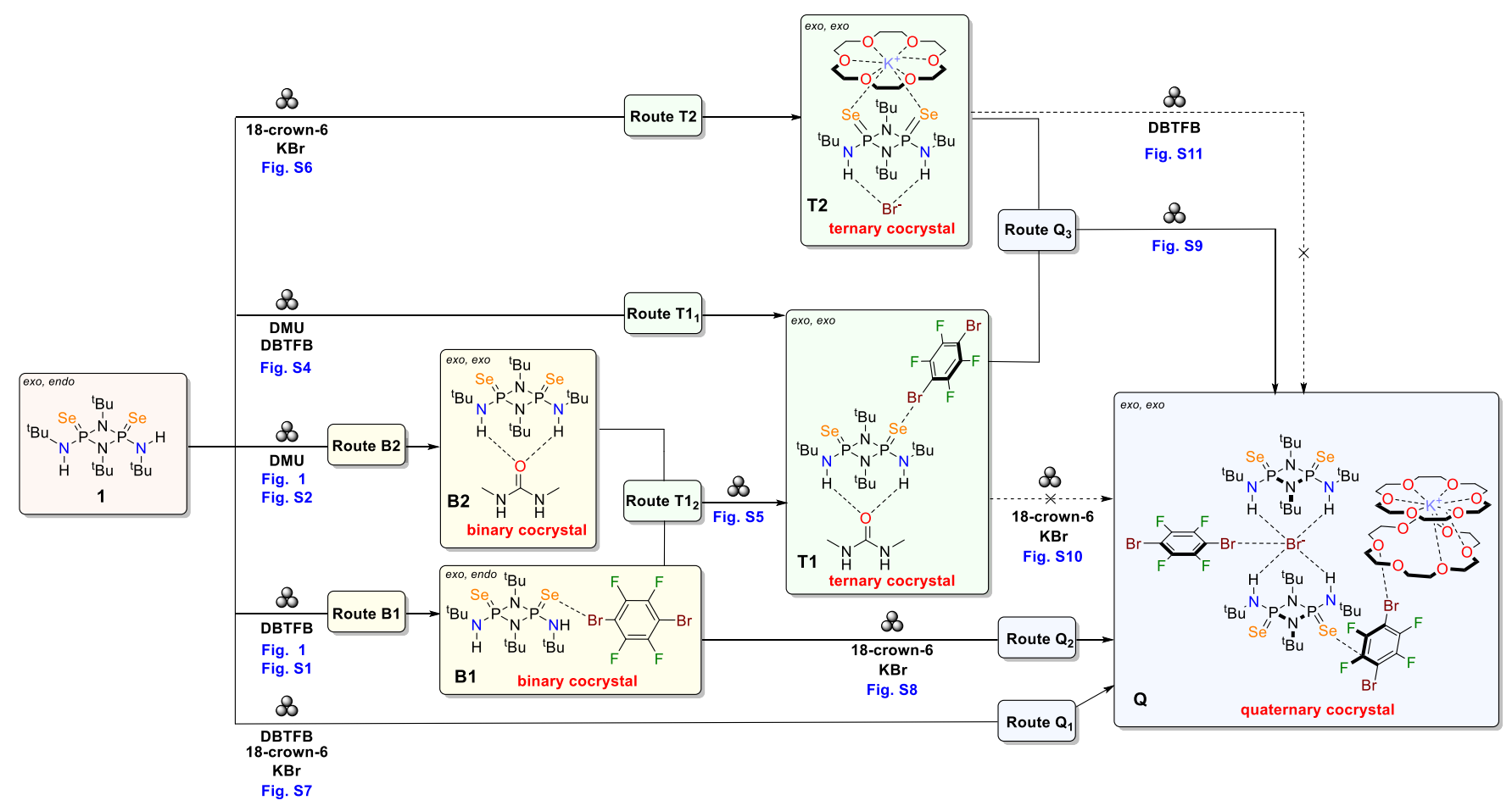

Scheme 2. Transformation and construction of multicomponent cocrystals based on the $\mathrm{Se}_{2} \mathrm{P}_{2} \mathrm{~N}_{2}$ phosphazane molecule (1) using mechanochemistry. The ternary ( $\mathbf{T} \mathbf{1}$ and $\mathbf{T} \mathbf{2})$ and quaternary cocrystal $(\mathbf{Q})$ can be obtained sequentially in neat grinding (Routes $\mathbf{T}_{\mathbf{2}}, \mathbf{Q}_{\mathbf{2}}$ and $\mathbf{Q}_{3}$ ) experiments or by direct one-pot neat grinding (Routes $\mathbf{T}_{\mathbf{1}}, \mathbf{T}_{\mathbf{2}}$ and $\mathbf{Q}_{\mathbf{1}}$ ) experiments from the individual components.

Our mechanochemical methodology ensures the rapid and sustainable synthesis of hybrid high-order organicinorganic cocrystals without any risk of solvent inclusion. Moreover, we also demonstrate that transformation and formation of ternary and quaternary cocrystals can be achieved by using lower-order binary and ternary cocrystals as reagents. Such synthetic versatility to achieve higher-order cocrystals, to the best of our knowledge is hitherto unreported. All obtained solid products were analyzed using X-Ray diffraction (single-crystal and powder diffraction) techniques to establish the identity of the compounds as well as the purity in the bulk samples.

In addition, density functional theory (DFT) methods were used to compute the thermodynamic driving force for the formation of the hybrid cocrystals and the mechanical properties of the resulting cocrystals were used to compare their bulk properties. The variations in the thermodynamic stabilization energies and computed mechanical properties of the cocrystals underscore the importance of carefully choosing the chemical building blocks used to target ternary and quaternary cocrystals.

This work has the potential to create new opportunities for the use of hybrid organic-inorganic molecular solids with specific, desirable and finely-tuned properties. Moreover, the results indicate that the synergistic application of mechanosynthesis and in-silico computational methods can provide an efficient and green technology for the rapid screening and selection of higher- order cocrystals, thereby allowing for the control of the functionality of the resulting hybrid cocrystals.

\section{Results and Discussion}

\section{Direct Synthesis of Binary Cocrystals (B1 and B2)}

In a previous study, it has been demonstrated that airand moisture-stable cyclodiphosph(V)azane compounds can be readily accessed via simple oxidation of the phosphorus(III) centers to phosphorus(V) using chalcogenic elements. ${ }^{[51-53]}$ Within the cyclodiphosph(V/V)azane species, the cis-oxidized frameworks the terminal substituents can adopt several topological arrangements giving rise to different conformations (Scheme 1). Of particular interest is the exo, exo $(\mathrm{Z}, \mathrm{Z})$ conformation, which acts as a versatile bifurcated hydrogen bond (HB) donor, owing to the two terminal $\mathrm{NH}$ groups that are oriented in a converging manner. Seminal work from Goldfuss et al. demonstrated that functionalized cis-oxidized cyclodiphosphazanes could be used for selective halide and acetate recognition via hydrogen-bonding with the two $\mathrm{NH}$ groups. ${ }^{[54]}$ This approach has recently been used in the synthesis of a range of versatile monomeric, ${ }^{[55]}$ dimeric and trimeric halide hosts. ${ }^{[32]}$

Additionally, we have demonstrated that the symmetrically substituted cis-Se oxidized cyclodiphosph(V/V)azane comprising four tert-butyl groups - $\left[{ }^{t} \mathrm{BuNH}\left(\mathrm{P}(\mathrm{Se}) \mu-\mathrm{N}^{t} \mathrm{Bu}\right)\right]_{2}, \quad\left(\mathbf{S e}_{2} \mathbf{P}_{2} \mathbf{N}_{2}\right)-$ can be cocrystallized with $\mathrm{N}, \mathrm{N}^{\prime}$-dimethylurea (DMU) and 1,4- 
dibromotetrafluorobenzene (DBTFB) in a 1:1 ratio to form

binary $\left(\mathbf{S e}_{2} \mathbf{P}_{2} \mathbf{N}_{2}\right) \cdot(\mathbf{D B T F B})$ and $\left(\mathbf{S e}_{2} \mathbf{P}_{2} \mathbf{N}_{2}\right) \cdot(\mathrm{DMU})$
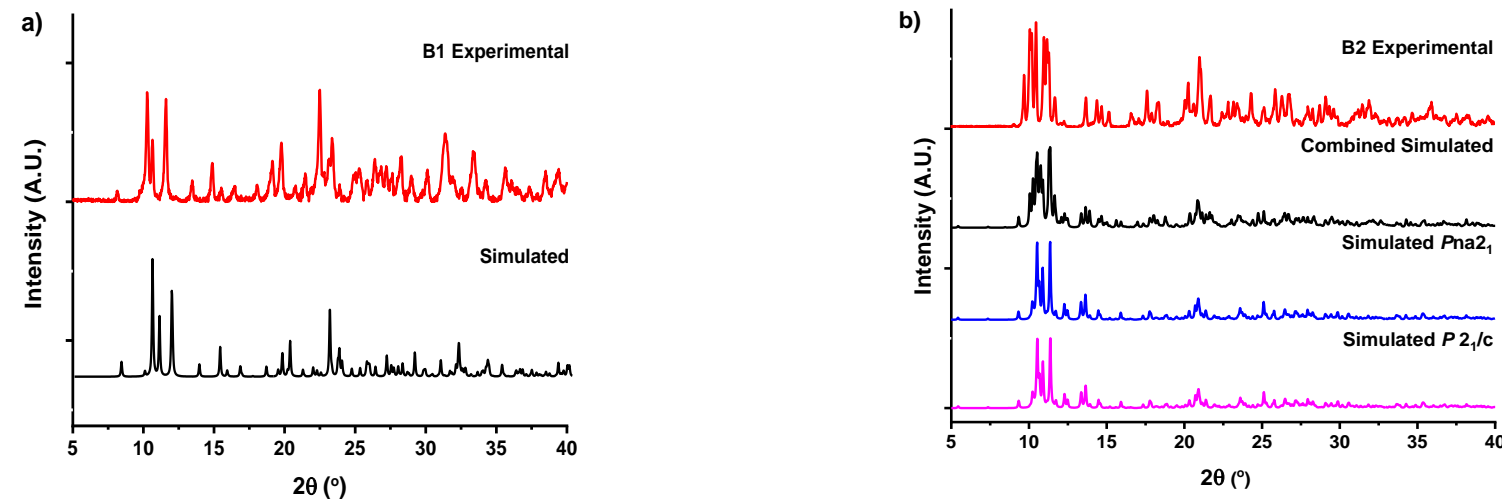

Figure 1. Overlay of powder diffraction patterns of the mechanochemically obtained powders and simulated patterns from single crystal data of the binary cocrystals of $\mathbf{S e}_{2} \mathbf{P}_{2} \mathbf{N}_{2}$ and a) DBTFB, b) DMU; two polymorphs of the binary cocrystal were obtained. For expanded view see SI

cocrystals (B1 and B2, respectively). ${ }^{[34]}$ Relevant to the herein reported results, we can highlight that the structure of $\mathbf{S e}_{2} \mathbf{P}_{2} \mathbf{N}_{2}$ (1, previously reported to adopt an exo, endo ( $Z$, $E$ ) conformation (CSD code XOTGAO) ${ }^{[56]}$ rearranges to an exo, exo $(Z, Z)$ conformation, $\mathbf{S e}_{2} \mathbf{P}_{2} \mathbf{N}_{2}{ }^{\text {exo,exo }}$ in Scheme $\mathbf{1}$, upon the formation of $\mathrm{R}^{2}{ }_{1}(8)$ bifurcated hydrogen bonds in B2. Binary cocrystal B2 can be isolated in two different polymorphic forms (i.e., B2-Form I and B2-Form II, Pnaz and $P_{2_{1}} / \mathrm{c}$, respectively). The two polymorphs differ in the number of symmetrically inequivalent molecules in the asymmetric unit with B2-Form I comprise 3 pairs of $\mathbf{S e}_{2} \mathbf{P}_{2} \mathbf{N}_{2}{ }^{[57]}$ and DMU (total 6 molecules) whilst $\mathbf{B 2}$-Form II comprises only $\mathbf{2}$ pairs of $\mathbf{S e}_{\mathbf{2}} \mathbf{P}_{\mathbf{2}} \mathbf{N}_{\mathbf{2}}$ and DMU (total 4 molecules). The packing index (PI) of Form I is $63.30 \%$, whilst Form II displays a PI of $65.00 \%$ (Table 1). Both polymorphs display solvent-accessible voids in the crystals (0.59\% and $3.74 \%$ of the unit cell volume in Form I and for Form II, respectively, see Table 1), as well as linear hydrogen-bonded chains comprising $\mathrm{R}_{1}{ }_{1}(8)$ bifurcated (N$\mathrm{H})_{2} \ldots \mathrm{O} \mathrm{HB}$ interactions in addition to an $\mathrm{R}_{2}{ }_{2}(10)$ nonbifurcated NH...Se interactions. While in polymorph B2Form $I$ the $\left(\mathbf{S e}_{2} \mathbf{P}_{2} \mathbf{N}_{2}\right) \cdot(\mathbf{D M U})$ units rotate by approximately $90^{\circ}$ along the chain, in B2-Form II we observe not only one but two $\left(\mathbf{S e}_{2} \mathbf{P}_{2} \mathbf{N}_{2}\right) \cdot(\mathbf{D M U})$ units connected by bis-bifurcated $(\mathrm{N}-\mathrm{H})_{2} \cdots$ Se interactions that rotate in an analogous manner (See SI).
In the $\mathbf{B} 1$ cocrystal, the $\mathbf{S e}_{2} \mathbf{P}_{2} \mathbf{N}_{\mathbf{2}}$ molecule adopts an exo, endo $(Z, E)$ conformation $\left(\mathbf{S e}_{2} \mathbf{P}_{\mathbf{2}} \mathbf{N}_{\mathbf{2}}\right.$ exo,endo, Scheme $\left.\mathbf{1}\right)$ instead - identical to the XOTGAO structure vide supra. This is attributed to the absence of strong hydrogen bond acceptors which is the driving force for adopting the exo, exo $(Z, Z)$ conformation. The asymmetric nature of $\mathbf{S e}_{2} \mathbf{P}_{2} \mathbf{N}_{2}{ }^{\text {eox,endo }}$ results in $\mathbf{B l}$ displaying halogen-bonding and $\mathrm{Se} \cdots \pi$ interactions with DBTFB molecules on the exo and endo sides, respectively (see $\mathbf{S I}$ ).

Hence in this study, we investigated the formation of these binary cocrystals using mechanochemistry. Neat grinding of reagents in a vibrational ball-mill produced microcrystalline powders of both binary cocrystals within $30 \mathrm{~min}$, as evidenced by powder X-ray diffraction (PXRD) patterns (Figure 1). Comparison of the diffractograms of the resultant powder with that of the individual components showed no trace of the starting materials (Figures S1-S2) and the pattern matched those simulated from single crystal diffraction data.

The successful mechanochemical synthesis of the binary cocrystals provided the motivation for the construction of our higher-order cocrystals. Hence, rational hierarchical intermolecular interactions and orthogonal synthons were judiciously combined to furnish higher-order ternary and quaternary cocrystals (vide infra). 
(a)

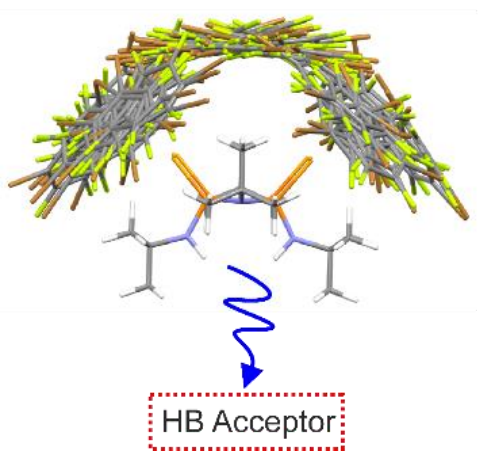

(b)

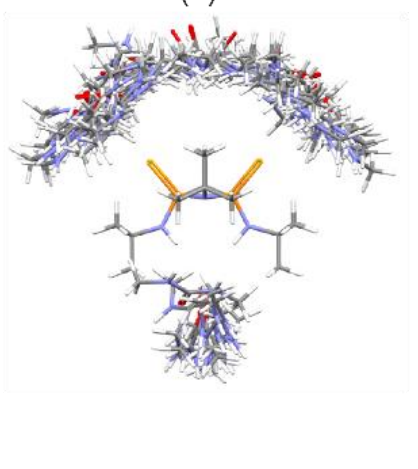

(c)

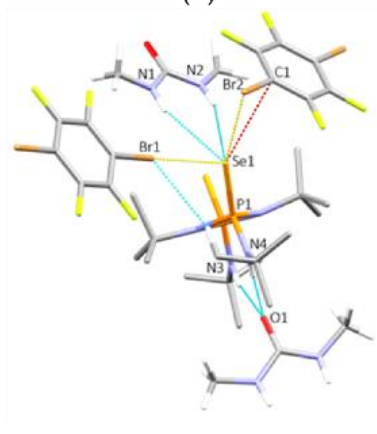

Figure 2. Monte-Carlo simulated trajectories for the 100 most stable binding geometries between $\mathbf{S e}_{2} \mathbf{P}_{2} \mathbf{N}_{2}$ exo,exo and either (a)

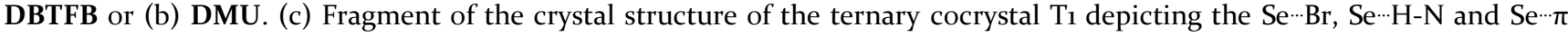
interactions. Some C-H hydrogens are omitted for clarity.

\section{Direct and Telescopic Mechanosynthesis of Ternary cocrystal 1 (T1): $\left(\mathrm{Se}_{2} \mathrm{P}_{2} \mathrm{~N}_{2}\right) \cdot(\mathrm{DMU}) \cdot(\mathrm{DBTFB})$.}

As $\mathbf{S e}_{2} \mathbf{P}_{2} \mathbf{N}_{2}$ can engage in both hydrogen and halogenbonding interactions at different sites (i.e., $\mathrm{NH}$ moieties and Se atoms, respectively), we attempted the supramolecular synthesis of ternary cocrystals involving $\mathrm{Se}_{2} \mathbf{P}_{2} \mathbf{N}_{2}$, DMU and DBTFB. This initial exercise is a proofof-concept of using synthon orthogonality as a crystal engineering tool to generate higher-order cocrystals, in such systems. This is also possible because of the bifunctionality of the cyclophosphazane molecule $\mathbf{S e}_{2} \mathbf{P}_{2} \mathbf{N}_{2}$ to be able to form halogen- (B1) and hydrogen-bonded (B2) binary cocrystals at different sites. Such a siteselective bifunctionality in engineering ternary cocrystals was first confirmed by initial Monte-carlo simulations. The calculations suggested that $\mathbf{S e}_{2} \mathbf{P}_{2} \mathbf{N}_{\mathbf{2}}$ can form binary complexes with DBTFB via Se $\cdots \pi$ or Se...Br interactions (Figure 2a), leaving the $\mathrm{NH}$ hydrogen bond donors on $\mathbf{S e}_{2} \mathbf{P}_{2} \mathbf{N}_{\mathbf{2}}$ available for engaging in intermolecular hydrogen bonding interactions with a suitable acceptor. Simulations involving DMU as the coformer also suggest that stable complexes with $\mathbf{S e}_{2} \mathbf{P}_{2} \mathbf{N}_{2}$ can be formed via the formation

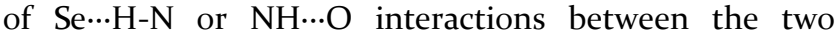
molecules (Figure 2b). Thus, we hypothesized that these synthons would not compete with one another (orthogonality) if both DBTFB and DMU were used in cocrystallization experiments with $\mathbf{S e}_{2} \mathbf{P}_{2} \mathbf{N}_{\mathbf{2}}$.

Indeed, milling Se2P2N2, DMU, and DBTFB in a 2:2:1 ratio at $30 \mathrm{~Hz}$ for $30 \mathrm{~min}$ - direct one-pot Route $\mathbf{T}_{\mathbf{1}_{\mathbf{1}}}$ in Scheme 2 - readily afforded a new molecular solid powder - with almost quantitative conversion of the individual components to the new crystalline product. This is evident from the PXRD patterns obtained (Figure $\mathbf{S}_{\mathbf{4}}$ ). Diffraction quality single crystals of the $\left(\mathbf{S e}_{2} \mathbf{P}_{2} \mathbf{N}_{2}\right) \cdot(\mathbf{D M U}) \cdot(\mathrm{DBTFB})$, (T1) neutral ternary cocrystal, were successfully obtained by slow evaporation of the methanol solution of the reaction mixture. The simulated PXRD pattern obtained from the single crystal data matched almost perfectly to those of the powders obtained mechanochemically. Analysis of the crystal structure of $\mathbf{T} \mathbf{1}$ revealed that the bifurcated hydrogen-bonding of $\mathbf{S e}_{2} \mathbf{P}_{2} \mathbf{N}_{\mathbf{2}}$ with DMU persists, along with Type II bifurcated halogen-bonding interactions via the ditopic DBTFB molecule (Figure 2c). $[58]$

There is a strong agreement between the dominant intermolecular forces obtained from the Monte Carlo simulations and the observed interactions in the experimental structure of T1. The preferential "top face" binding of DBTFB on the of $\mathrm{Se}_{2} \mathrm{P}_{2} \mathrm{~N}_{2}$ in all the Monte Carlo binding trajectories ensures that only $\mathrm{Se} \cdots \pi$ or $\mathrm{Se} \cdots \mathrm{Br}$ interactions are observed between these molecules in $\mathbf{T}$. Consequently, the oxygen of DMU is able to engage in a bifurcated hydrogen bond with the unused $\mathrm{NH}$ donors of $\mathrm{Se}_{2} \mathrm{P}_{2} \mathrm{~N}_{2}$.

Such orthogonal interplay between hydrogen- and halogen-bonding is reminiscent of a related system involving thioureas. ${ }^{[28]}$ However, in this ternary cocrystal,

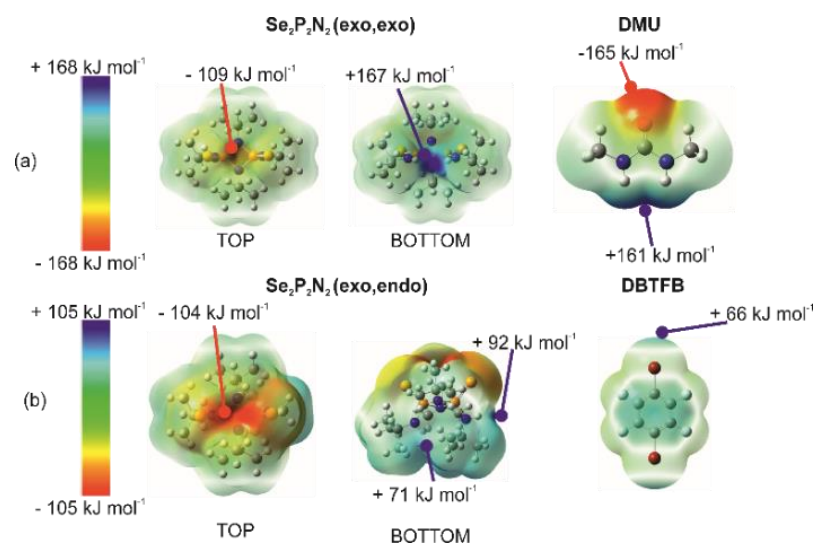

Figure 3. Molecular electrostatic potential (MEP) surfaces for (a) $\mathbf{S e}_{2} \mathbf{P}_{2} \mathbf{N}_{\mathbf{2}}$ (top and bottom views) and DMU; (b) $\mathbf{S e}_{\mathbf{2}} \mathbf{P}_{2} \mathbf{N}_{\mathbf{2}}$ (top and bottom views) and DBTFB. See SI for expanded version.

one of the Se atoms of the $\mathbf{S e}_{2} \mathbf{P}_{2} \mathbf{N}_{\mathbf{2}}$ can simultaneously undergo three different types of intermolecular interactions, a halogen bond to a molecule of DBTFB (Se $\cdots$ Br distance $c a$. $3.42 \AA$ ), a hydrogen-bond acceptor for the bifurcated hydrogen-bonds of DMU ( $\mathrm{Se} \cdot \cdots \mathrm{H}-\mathrm{N}$ distance 
ca. $3 \cdot 71 \AA$ ), and a long range Se- $\pi$ interaction to the electron deficient ring of another adjacent DBTFB molecule (Se $\cdots \mathrm{C}$ distance ca. $3.43 \AA$ ) - also observed in the B1 binary cocrystal.

Notably, the ability of Se atoms to accommodate several different supramolecular interactions has rarely been observed, and to the best of our knowledge, has not been previously reported. This can be attributed to the relatively large size of the Se atom (ca. 1.64 $\AA^{3}$ ) and the electron rich cyclodiphosphazane $\mathrm{P}_{2} \mathrm{~N}_{2}$ backbone whose nitrogen lone pairs can delocalize and conjugate onto the $\mathrm{P}=$ Se moiety, enabling it to undergo halogen- and hydrogen-bonding, as well as Se $\cdots \pi$ interactions. This further highlights the unique nature of Se-oxidized cyclodiphosphazanes as inorganic building blocks to construct complex supramolecular assemblies.

Whilst the formation of binary cocrystals via mechanochemistry has previously been described, ${ }^{[42,54,55]}$ the factors dictating the transformation of these binary crystals into higher-order ternary and quaternary crystals is poorly understood. Hence, the obtained cyclodiphosphazane-based ternary cocrystal (T1) presents a unique opportunity to provide proof-of-concept data as to whether ball-milling can be used to construct higherorder cocrystals using a lower-order system (i.e., binary crystals) as supramolecular building blocks - which to be best of our knowledge has never been demonstrated. It is important to highlight that the use of binary cocrystals as reagents to form higher-order cocrystals cannot be achieved in solution, but can be realized mechanochemically in the solid-state. This further highlights the efficacy and practicality of using mechanochemistry as a powerful synthetic tool to generate complex multicomponent solids.

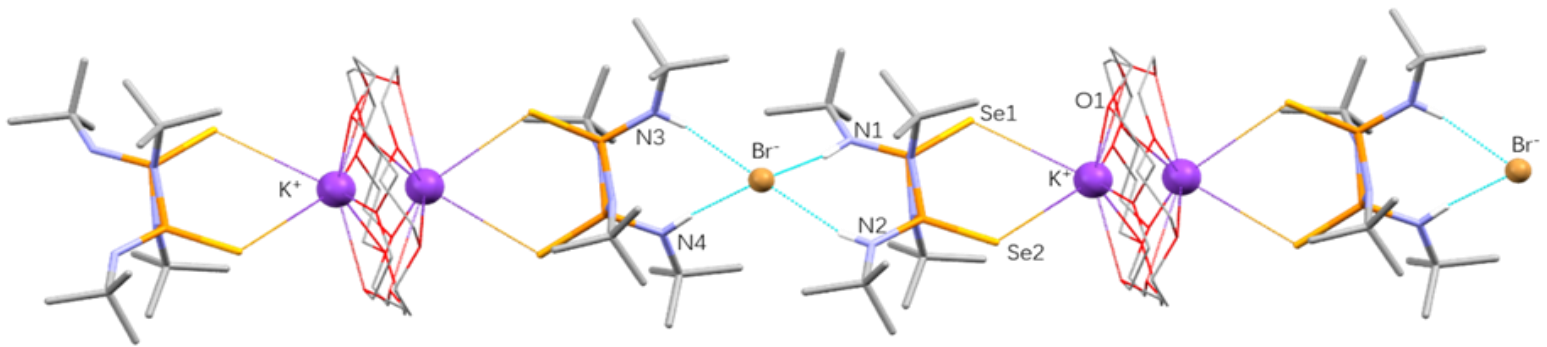

Figure 4. Fragment of the crystal structure of the ternary ionic cocrystal $\left(\mathbf{S e}_{\mathbf{2}} \mathbf{P}_{\mathbf{2}} \mathbf{N}_{\mathbf{2}}\right) \cdot(\mathbf{c r o w n}) \cdot(\mathrm{KBr}) \mathbf{T} \mathbf{2}$ forming one-dimensional chains linked by $\mathrm{Se}^{\cdots} \mathrm{K}^{+}$and $\mathrm{Br}^{-\cdots} \mathrm{H}-\mathrm{N}$ interactions. The crown and $\mathrm{K}^{+}$are positionally disordered over two sites. Some $\mathrm{C}-\mathrm{H}$ hydrogens are omitted for clarity. For expanded version see SI.

Our results suggest that Ti can be successfully obtained by combining binary cocrystals of $\mathbf{B 1}$ and $\mathbf{B 2}$ in equimolar amounts - sequential Route $\mathbf{T r}_{\mathbf{2}}$ in Scheme 2 (see Figure $\mathbf{S}_{\mathbf{5}}$ ). The successful synthesis of the T1 ternary cocrystal from either binary cocrystal precursors or individual components demonstrates the functional flexibility and potential of $\mathbf{S e}_{\mathbf{2}} \mathbf{P}_{\mathbf{2}} \mathbf{N}_{\mathbf{2}}$ to form robust and reproducible supramolecular synthons.

The calculated molecular electrostatic potentials (MEPs, Figure 3) support the observed heterosynthons in the cocrystals. DMU engages in directional HB interactions with $\mathbf{S e}_{\mathbf{2}} \mathbf{P}_{\mathbf{2}} \mathbf{N}_{\mathbf{2}}$ in $\mathbf{B}_{2}$ and $\mathbf{T}_{\mathbf{1}}$ because of the large negative potential (i.e., $-165 \mathrm{~kJ} \mathrm{~mol}^{-1}$ ) on the carbonyl acceptor group. By contrast, the bromines on DBTFB are associated with a positive potential of $+66 \mathrm{~kJ} \mathrm{~mol}^{-1}$, thereby facilitating the formation of complementary Se $\cdots \mathrm{Br}$ interactions in $\mathbf{B}_{\mathbf{1}}$ and T1. For the $\mathbf{S e}_{\mathbf{2}} \mathbf{P}_{2} \mathbf{N}_{\mathbf{2}}$ molecules, the observed electron density distribution is strongly dependent on the conformation of the molecule. The exo,exo conformation has a strong positive potential of approximately $+167 \mathrm{~kJ} \mathrm{~mol}^{-1}$ due to converging $\mathrm{NH}$ donors, which are oriented in the same direction. By contrast, the exo,endo conformation is a weaker hydrogen bond donor as indicated by the comparable potentials of $+71 \mathrm{~kJ} \mathrm{~mol}^{-1}$ and $+92 \mathrm{~kJ} \mathrm{~mol}^{-1}$ at each NH donor site (Figure 3).

In line with previous reports, ${ }^{[55-56]}$ this variation in the hydrogen bond donor capacity of $\mathbf{S e}_{\mathbf{2}} \mathbf{P}_{\mathbf{2}} \mathbf{N}_{\mathbf{2}}$ explains why we observe $\mathbf{S e}_{2} \mathbf{P}_{2} \mathbf{N}_{\mathbf{2}}$ only in the (exo,exo) conformation in all cocrystals containing DMU (B2-Form I, B2-Form II and T1) since it is the only conformation which has NH donors that are able to engage in strong directional bifurcated hydrogen bonding interactions with the oxygen acceptor of DMU. The calculated MEPs also support the observed orthogonality of hydrogen- and halogen-bonding interactions in ternary cocrystal T1. This is evident from the MEPs as the NH donors on $\mathbf{S e}_{\mathbf{2}} \mathbf{P}_{\mathbf{2}} \mathbf{N}_{\mathbf{2}}$ are more likely to be engaged in hydrogen bonding interactions with the carbonyl acceptor of DMU since this intermolecular interaction has the strongest pairing according to the calculated potentials. This allows the Se atoms to freely engage in Se...Br interactions with DBTFB.

\section{Direct Synthesis of Ternary cocrystal 2 (T2): $\left(\mathrm{Se}_{2} \mathrm{P}_{2} \mathrm{~N}_{2}\right) \cdot(\mathrm{KBr}) \cdot(18-\mathrm{crown}-6$ ether)}

As previously described, the development of twocomponent molecular salts has been established in the area of pharmaceutical solids as a means to enhance the physicochemical properties of APIs. ${ }^{[61-63]}$ However, progress in making ICCs with three- or more molecular components is still lacking. ${ }^{[29,64]}$ In the context of species comprising complex main group ionic species, such ICCs resemble the inverse coordination of salt lattices by metal phosphide complexes. ${ }^{[65-65]}$. 
We hypothesize that the proven ability of cyclodiphospha(V/V)zanes frameworks to act both as ligands to coordinate metal cations via the formation of SeM coordinative bonds, ${ }^{[66-68]}$ and their already reported ability to bind to halides via bifurcated hydrogen bonding ${ }^{[69]}$ would enable higher-order ICC cocrystals. We envisioned the formation of ternary $\mathrm{ICC}^{\left[{ }^{[70-73]}\right.}$ with the combination of $\mathbf{S e}_{\mathbf{2}} \mathbf{P}_{\mathbf{2}} \mathbf{N}_{\mathbf{2}}$ with simple ionic salts such as $\mathrm{KBr}$ via Se-cation and $\mathrm{N}-\mathrm{H} \cdot \cdots \mathrm{X}^{-}$interactions (Figure 4).

Initial screening experiments by grinding $\mathbf{S e}_{\mathbf{2}} \mathbf{P}_{\mathbf{2}} \mathbf{N}_{\mathbf{2}}$ with $\mathrm{KBr}$, however, did not result in any changes of the powder pattern, suggesting the formation of a mere physical mixture (Figure S3). Hence, 18-crown-6 ether (crown) was added, a commonly used strategy in organic chemistry for trapping $\mathrm{K}^{+}$(and other alkali) metal cations. ${ }^{\left[{ }^{[7-76]}\right.}$ Under optimized conditions, milling $\mathbf{S e}_{2} \mathbf{P}_{2} \mathbf{N}_{2}$, crown and $\mathbf{K B r}$, in a 2:1:1 molar ratio for three hours - direct one-pot Route

a)

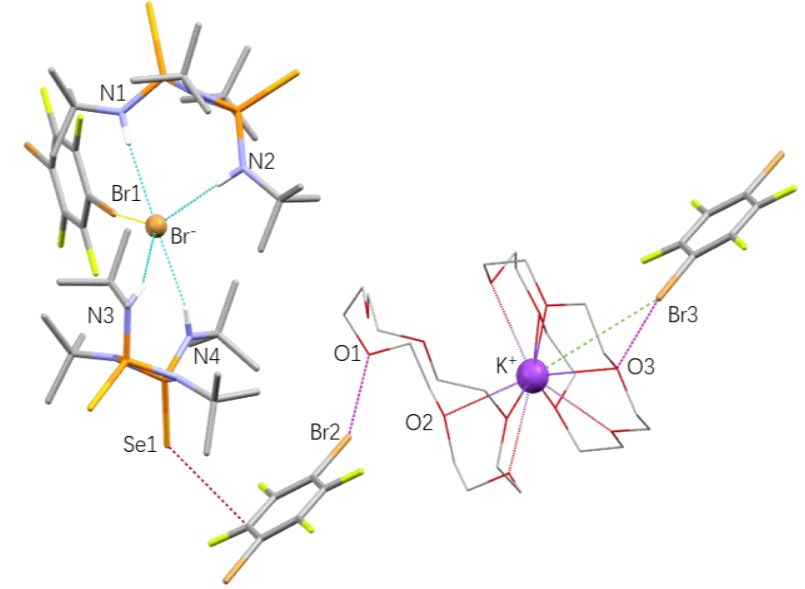

T2 in Scheme 2 - showed the diffraction peaks corresponding to the starting materials rapidly disappear and a new PXRD pattern was observed (Figure S6). The new pattern suggests the successful formation of the desired $\left(\mathbf{S e}_{2} \mathbf{P}_{\mathbf{2}} \mathbf{N}_{\mathbf{2}}\right) \cdot($ crown $) \cdot(\mathbf{K B r}) \quad\left(\mathbf{T}_{2}\right)$ ionic cocrystal. Diffraction quality crystals of $\mathbf{T}_{\mathbf{2}}$ were subsequently obtained by slow evaporation from solution using either $\mathrm{CHCl}_{3}$ or isopropanol as the solvent.

The crystal structure of $\mathbf{T} \mathbf{2}$ comprises a one-dimensional catemer where the $\mathrm{K}^{+}$cation is coordinated to a crown ether moiety and a $\mathbf{S e}_{\mathbf{2}} \mathbf{P}_{\mathbf{2}} \mathbf{N}_{\mathbf{2}}$ unit acting as a chelating ligand through the $\mathrm{P}=\mathrm{Se}$ bonds $\left(\mathrm{Se} \cdot \cdots \mathrm{K}^{+} \quad 3.75 \AA\right)^{[34,75]}$. The complexed $\mathrm{K}^{+}$crown portion is disordered over two sites along the chains. On the other hand, the $\mathrm{Br}^{-}$counter anion is stabilized by two sets of bifurcated charge-assisted hydrogen-bonds (CAHyB) via four $\mathrm{N}-\mathrm{H}$ moieties from two $\mathbf{S e}_{2} \mathbf{P}_{2} \mathbf{N}_{2}$ molecules $\left(\mathrm{N}-\mathrm{H} \cdots \mathrm{Br}^{-} 3.40 \AA\right)^{[77]}$ within this chain.

b)

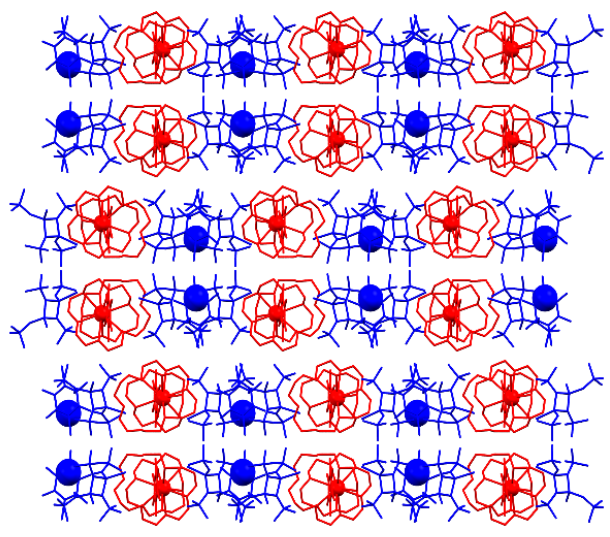

Figure 5. a) Fragment of the crystal structure of the four-component ionic cocrystal $\left(\mathrm{Se}_{2} \mathrm{P}_{2} \mathrm{~N}_{2}\right)_{2} \cdot(\mathrm{DBTFB})_{2} \cdot(\mathrm{crown})_{2} \cdot(\mathrm{KBr})(\mathrm{Q})$ depicting the $\mathrm{N}-\mathrm{H}^{\prime} \cdots \mathrm{Br}^{-}, \mathrm{O} \cdots \mathrm{Br}, \mathrm{Br}^{\cdots} \mathrm{Br}^{-}, \mathrm{O} \cdots \mathrm{K}^{+}$and $\mathrm{Se} \cdots \pi$ interactions; b) Fragment of the crystal structure of the four-component ionic cocrystal Q depicting the packing motif as viewed down the crystallographic c-axis. The red and blue domains are linked by $\mathrm{Se} \cdots \pi$ interactions. The blue spheres are the $\mathrm{Br}^{-}$anions and the red spheres are the $\mathrm{K}^{+}$cations. For expanded version see SI.

\section{Direct and Telescopic Synthesis of Quaternary cocrystal (Q)}

The formation of quaternary and other higher-order cocrystals can be very challenging, ${ }^{[20]}$ especially in systems involving a large variety of functionalities that can form different combinations of intermolecular interactions, rendering the prediction of any supramolecular synthon hierarchy challenging. ${ }^{[73-74]}$ Therefore, we endeavored to explore the synthesis of higher-order quaternary cocrystals using our mechanochemical approach, supported by computational modelling (vide infra).

Ball-milling $\mathrm{Se}_{2} \mathbf{P}_{2} \mathbf{N}_{2}$, DBTFB, crown and $\mathrm{KBr}$ in 2:2:2:1 and 2:2:1:1 molar ratios for $30 \mathrm{~min}$ at $30 \mathrm{~Hz}$ - direct one-pot Route $Q_{1}$ in Scheme 2 - resulted in the formation a microcrystalline powder, whose PXRD pattern contained new peaks at low angles which indicate a new solid form containing a large unit cell (Figure $S_{7}$ ) had formed. Diffraction quality crystals were obtained by slow crystallization of the powder mixture from THF, which were analyzed by SCXRD (Figure 4).
Crystal structure analysis confirmed the successful formation of a four-component ICC $\left(\mathrm{Se}_{2} \mathrm{P}_{2} \mathrm{~N}_{2}\right)_{2} \cdot(\mathrm{DBTFB})_{2} \cdot(\text { crown })_{2} \cdot(\mathrm{KBr}), \quad(\mathbf{Q}$ in Figure 5$)$. The quaternary ICC revealed several intermolecular interactions, which provide insights into the supramolecular hierarchy within our system. Firstly, the robust bifurcated hydrogen-bonding synthon of the $\mathbf{S e}_{2} \mathbf{P}_{2} \mathbf{N}_{2}$ was retained, and similar to the $\mathbf{T}_{\mathbf{2}}$ cocrystal, the $\mathrm{Br}^{-}$is stabilized by four CAHyB $\mathrm{N}-\mathrm{H}^{-\cdots} \mathrm{Br}^{-}$interactions via two $\mathbf{S e}_{2} \mathbf{P}_{2} \mathbf{N}_{\mathbf{2}}$ molecules. Closer inspection of the intermolecular interactions revealed that the $\mathrm{Br}^{-}$also undergoes Type I halogen-bonding ${ }^{[73-74]}$ with a molecule of DBTFB. Such a $\mathrm{Br}^{-\cdots \mathrm{Br}^{-}}$interaction is reminiscent of charge-assisted halogen-bonds (CAHaB) that was previously observed by several groups. ${ }^{\text {[75] }}$

Another expected interaction is the formation of iondipole interactions between $\mathrm{K}^{+}$and two crown molecules, albeit one of the crown molecules not fully encapsulating the cation. Consequently, the formation of this "half- 
crown" complex creates coordinately available ether sites, allowing for $\mathrm{O} \cdots \mathrm{Br}$ halogen-bonds (2.94-2.99 $\AA$ ) to form with neighboring DBTFB molecules. The ditopic DBTFB molecules and the bis-crown- $\mathrm{K}^{+}$complex formed linear catemers linked by bidirectional halogen-bonds. The presence of strong $\mathrm{O} \cdots \mathrm{Br}$ halogen bonds could also explain the absence of the weaker Se.*Br halogen bond (ca. 3.42 $\AA$ ) in this cocrystal, illustrating the preferential hierarchy of $\mathrm{O} \cdots \mathrm{Br}$ vs $\mathrm{Se} \cdots \mathrm{Br}$ supramolecular interactions. ${ }^{\left[{ }^{22}\right]}$ However, despite the lack of $\mathrm{Se} \cdots \mathrm{Br}$ halogen bonds, the two crystallographically inequivalent DBTFB molecules still form Se $\cdots \pi$ interactions with the $\operatorname{Se}_{2} \mathbf{P}_{2} \mathbf{N}_{2}$ molecules present. This Se $\cdots \pi$ interactions (ca. 3.48-3.57 $\AA$ ) have also been observed in all cocrystals involving $\mathbf{S e}_{2} \mathbf{P}_{2} \mathbf{N}_{2}$ and DBTFB (two-, three- and four-component crystals), indicating their robustness and high reproducibility as supramolecular synthons.
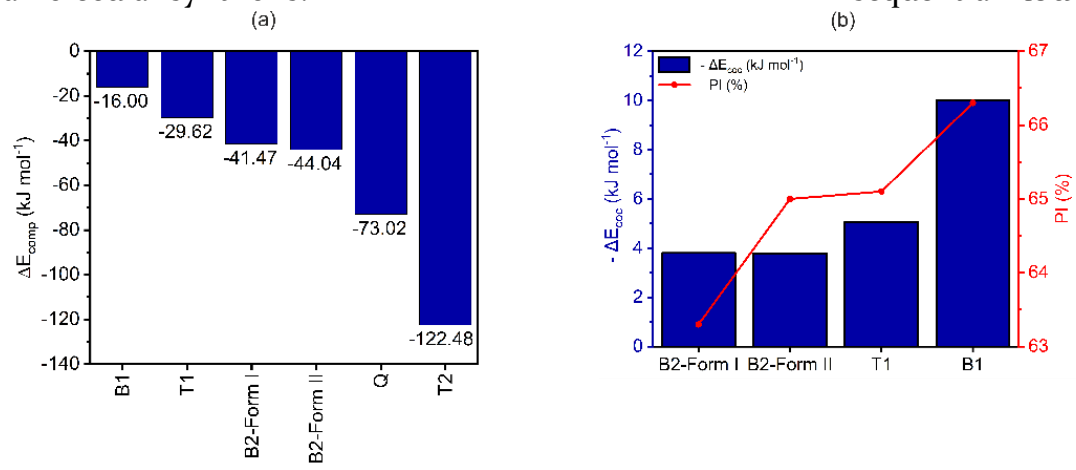

The obtained four-component ICC can be divided into two distinct domains (Figure $\mathbf{5} \mathbf{b}$ ). The first domain (in red) consists of a $\mathrm{Br}^{-}$anion with two molecules of $\mathbf{S e}_{\mathbf{2}} \mathbf{P}_{\mathbf{2}} \mathbf{N}_{\mathbf{2}}$ and one DBTFB molecule, stabilized by $\mathrm{CAHyB}$ and $\mathrm{CAHaB}$ interactions, respectively. The second domain (in blue) is composed of the $\mathrm{K}^{+}$cation encapsulated by two crown molecules, which formed halogen-bonded chains with another molecule of DBTFB. Both domains form alternating layers and are linked together by the two Se $\cdots \pi$ intermolecular interactions as they propagate along the crystallographic c-axis.

In order to further test our novel stepwise methodology for the synthesis of higher-order cocrystals, several alternative routes were explored for the preparation of the quaternary cocrystal (Q). Firstly, milling a mixture of the previously obtained binary cocrystal $\mathbf{B 1}$, crown and $\mathbf{K B r}-$ sequential Route $\mathbf{Q}_{2}$ - successfully produced the target

Figure 6: (a) Bar graph of the trend in the complexation energy $\left(\Delta \mathrm{E}_{\text {comp }}\right)$ for each cocrystal. (b) Variation in the DFT energy for the formation of the molecular cocrystals $\left(-\Delta \mathrm{E}_{\mathrm{coc}}\right) \mathbf{B} \mathbf{1}, \mathbf{B} \mathbf{2}$ and T1 with the packing index (PI) of each cocrystal. (c) Illustration of the observed synthons and relative strengths of the intermolecular interactions observed in the various cocrystals. For expanded version see SI.

quaternary cocrystal $\mathbf{Q}$ as indicated by the PXRD pattern of the obtained crystalline solid (Figure S8). Notably, attempts to apply the same protocol starting from the ternary cocrystals $\mathbf{T} \mathbf{1}$ and $\mathbf{T} \mathbf{2}$ did not produce the desired quaternary cocrystal $\mathbf{Q}$ (see Scheme 2 and Figures S1oS11), although ongoing efforts are being made to optimize these two routes. Finally, the stepwise approach was also tested for the synthesis of the four-component ionic cocrystal by milling together both ternary cocrystals T1 and T2 as precursors. Interestingly, the milling of both ternary cocrystals $\mathbf{T} 1$ and $\mathbf{T}_{2}$ in a 1:1 ratio - sequential Route $\mathbf{Q}_{3}$ in Scheme 2- yielded the quaternary cocrystal $\mathbf{Q}$ as indicated by the PXRD of the obtained crystalline powder (Figure S9).

Overall, binary, ternary and quaternary cocrystals based on the $\mathrm{Se}_{2} \mathrm{P}_{2} \mathrm{~N}_{2}$ molecule had been successfully obtained. These species can be made from their single individual components, or lower-order (i.e., binary and ternary) crystal building blocks in both a one-pot and sequential stepwise manner. Demonstration of telescopic supramolecular transformations involving more than two components, without solvate formation, is rare and reported examples are few and far between. [45-48] Such synthetic versatility in a mechanochemical reaction is reminiscent of a similar report by Hernandez et al., that involved transformation of organometallic $\operatorname{Re}$ compounds. ${ }^{[81]}$

Energetic considerations for crystallizing higher-order cocrystals and comparison of the predicted mechanical properties

The systematic crystal engineering of higher-order cocrystals requires a fundamental understanding of the driving forces behind their formation. Generally, the formation of binary cocrystals is thermodynamically driven. This has recently been verified in an extensive survey of the energetics of forming 350 binary cocrystals comprising both hydrogen and halogen bonding interactions. ${ }^{\left[8_{2}\right]}$ On average, more than $95 \%$ of the surveyed cocrystals were more stable than the stoichiometrically weighted sum of their component crystals. The average stabilization energy for the set of binary cocrystals surveyed was $-8 \mathrm{~kJ} \mathrm{~mol}^{-1}$. In contrast, for higher-order cocrystals such as $\mathbf{T} \mathbf{1}, \mathbf{T} \mathbf{2}$ and $\mathbf{Q}$ much less is known about the energetics behind their formation.

Recent work on hydrogen-bonded ternary molecular ICCs ${ }^{\left[8_{3}\right]}$ has shown that their crystal structures can be reliably predicted using computational methods ${ }^{[84]}$ due to the presence of predictable $\mathrm{CAHyB}$ interactions within 
these systems. However, the mean stabilization energy for these ternary cocrystals was found to only be $-2 \mathrm{~kJ}$ mol-1, which is significantly less stable than that observed for binary cocrystals. ${ }^{\left[{ }^{8}\right]}$ This suggests that factors other than thermodynamics may be important in determining which set of binary cocrystals can be used to construct ternary and quaternary cocrystals.

In an attempt to understand the energetics of forming the diverse set of cocrystals discovered in this work (Table 1), two different types of DFT calculations were performed: (i) complexation energies $\left(\Delta \mathrm{E}_{\text {comp }}\right)$ between the unique chemical species in the crystals ignoring packing effects and where possible, (ii) bulk cocrystal $\left(\Delta \mathrm{E}_{\mathrm{coc}}\right)$ stabilization energies taking into account the crystal packing forces.

The computed complexation energies show a clear thermodynamic driving force for molecular association between the components in each cocrystal. The complexation energies $\left(\Delta \mathrm{E}_{\mathrm{comp}}\right)$ range from $-16 \mathrm{~kJ} \mathrm{~mol}^{-1}$ for B1 to $-122 \mathrm{~kJ} \mathrm{~mol}^{-1}$ for T2 (see Figure 6a and Table 1 ).

Moreover, there is a general trend of increasing stabilization going from binary to quaternary cocrystals, which agrees with the hierarchy of supramolecular synthons. Cocrystal $\mathbf{B} 1$ displays the least stabilizing complexation energy due to the $\mathrm{Se} \cdot \cdots \mathrm{Br}$ heterosynthons being weaker than the $\mathrm{NH} \cdots \mathrm{O}=\mathrm{C}$ hydrogen bonds observed in both $\mathbf{B 2}$ and $\mathbf{T} \mathbf{1}$ (Figure 6a and $\mathbf{6 c}$ ). By contrast, T2 displays the most stabilizing complexation energy of all the cocrystals due to the favorable multi-center coulombic interactions in this ionic cocrystal. This is evidenced by the $\mathrm{K}^{+}$metal ions effectively coordinating to both $\mathbf{S e}_{\mathbf{2}} \mathbf{P}_{\mathbf{2}} \mathbf{N}_{\mathbf{2}}$ and the crown ether, leading to more stabilizing complexation energies in $\mathbf{T}_{\mathbf{2}}$ when compared to $\mathbf{Q}$ where the $\mathbf{S e}_{\mathbf{2}} \mathbf{P}_{\mathbf{2}} \mathbf{N}_{\mathbf{2}}$ molecules are not coordinated around the $\mathrm{K}^{+}$ions.

Table 1: Properties of the ICCs reported in this work. Packing index PI (\%), $\mathrm{V}_{\text {void }}=$ Void cell volume (\%), B=Bulk modulus, $\beta=$ Compressibility, $\Delta \mathrm{E}_{\text {comp }}=\mathrm{DFT}$ complexation energy per fragment in the asymmetric unit, $\Delta \mathrm{E}_{\mathrm{coc}}=\mathrm{DFT}$ bulk cocrystal stabilization energy per formula unit per formula unit.

\begin{tabular}{|c|c|c|c|c|c|c|c|c|}
\hline Solid Form & $\begin{array}{c}\mathrm{Se}_{2} \mathrm{P}_{2} \mathrm{~N}_{2} \\
\text { Conformatio } \\
n\end{array}$ & $\begin{array}{l}\text { Crystal } \\
\text { density } \\
\left(\mathrm{g} \mathrm{cm}^{-3}\right)\end{array}$ & PI (\%) & $\mathrm{V}_{\text {void }}(\%)$ & $\begin{array}{l}\Delta \mathrm{E}_{\text {comp }} / \\
\mathrm{kJ} \mathrm{mol}^{-1}\end{array}$ & $\begin{array}{c}\Delta \mathrm{E}_{\text {coc }} / \mathrm{kJ} \\
\mathbf{m o l}^{-1}\end{array}$ & B (GPA) & $\beta\left(\mathrm{TPa}^{-1}\right)$ \\
\hline B1 & exo-endo & 1.73 & 66.30 & 0.00 & -16.00 & -10.00 & 22.78 & 46.20 \\
\hline $\begin{array}{c}\text { B2-Form II } \\
\left(\mathrm{Z}^{\prime \prime}=4\right)\end{array}$ & exo-exo & 1.38 & 65.00 & 0.59 & -44.04 & -3.79 & 13.88 & 72.53 \\
\hline $\begin{array}{c}\text { B2-Form I } \\
\quad\left(Z^{\prime \prime}=6\right)\end{array}$ & exo-exo & 1.34 & 63.30 & 3.74 & -41.47 & -3.80 & $9 \cdot 31$ & 120.46 \\
\hline T1 & exo-exo & 1.51 & 65.10 & $3 \cdot 30$ & -29.62 & -5.07 & 9.01 & 141.58 \\
\hline $\mathbf{T} 2$ & exo-exo & 1.73 & 71.40 & 0.96 & -122.48 & - & - & - \\
\hline $\mathbf{Q}$ & exo-exo & 1.62 & 68.40 & 0.00 & -73.02 & - & - & - \\
\hline
\end{tabular}

Note: For $\mathbf{T} 2$ and $\mathbf{Q}$, the $\Delta \mathrm{E}_{\text {coc }}$, the bulk modulus (B) and compressibility $(\beta)$ could not be computed due to the lack of either reference structures $\left(\Delta \mathrm{E}_{\mathrm{coc}}\right)$ or suitable potentials $(\mathrm{B}$ and $\beta)$ for computing these properties.

When crystal packing effects are considered, DFT estimates of $\Delta \mathrm{E}_{\mathrm{coc}}$ (Table 1) suggest that the efficiently packed B1 cocrystal (PI: 66.30\%) displays the highest stabilization energy (Figure $6 \mathbf{b}$ and Table 1 ) of $-10 \mathrm{~kJ} \mathrm{~mol}^{-}$ ${ }^{1}$ relative to the stoichiometrically weighted sum of the component crystal energies. By contrast, the two polymorphs of B2 (i.e., B2-Form I and-Form II, Z" $=6$ and 4 respectively) are equi-energetic $(-3.80 \mathrm{~kJ} / \mathrm{mol})$ when crystal packing forces are considered and there is no discernible energetic preference for crystallizing one form over another (Figure 6b). We attribute this to the comparable packing forces of the molecules in the two forms as they display the same interactions between DMU and $\mathbf{S e}_{\mathbf{2}} \mathbf{P}_{\mathbf{2}} \mathbf{N}_{\mathbf{2}}$ but differ only in the number of symmetrically inequivalent units of $\mathbf{D M U}$ and $\mathbf{S e}_{2} \mathbf{P}_{2} \mathbf{N}_{2}$ in the crystal. When compared to B1, the two polymorphs of $\mathbf{B} 2$ display the lowest PI of all the cocrystals synthesized. The molecular ICC $\mathbf{T} \mathbf{1}$ is also predicted to be thermodynamically favored to form (Figure $\mathbf{6 b}$ and Table 1), according to the calculated $\Delta \mathrm{E}_{\mathrm{coc}}$ of $-5.07 \mathrm{~kJ} \mathrm{~mol}^{-1}$. For $\mathrm{T}_{2}$ and $\mathbf{Q}$, the absence of reference component crystal structures prevented computing $\Delta \mathrm{E}_{\text {coc. }}$

One of the key motivations for the discovery of higherorder cocrystals is to widen the range of solid-state properties that may be adopted by a reference molecule when formulated in different solid forms. This is possible because changing the chemical composition of crystals is known to affect the resulting bulk material properties. ${ }^{[85]}$ Mechanical properties are an example of a solid-state property that are critical to the performance characteristics of solid materials. In an attempt to understand how the cocrystal stoichiometry and observed intermolecular forces affect the mechanical properties of the solids, we computed the bulk modulus (B) and compressibilities ( $\beta$ ) for the cocrystals (Table 1). The predicted mechanical properties of the cocrystals vary significantly, depending on the types of interactions present (see Table 1). The halogen-bonded B1 cocrystal displays a bulk modulus of 22.78 GPa, which contrasts with the $13.88 \mathrm{GPa}$ predicted for the hydrogen-bonded Bz-Form II crystal. The less 
efficiently packed B2-Form I polymorph displays a bulk modulus of 9.31 GPa whilst Ti displays a bulk modulus of 9.01 GPa. The computed compressibilities are correlated with the packing efficiencies of the components in the crystals. B2-Form I and T1 both display large $\beta$ values that are $>100 \mathrm{TPa}^{-1}$, reflecting the poorly packed molecules in the cocrystals with void space accounting for more than $3 \%$ of the unit cell volumes. By contrast, the efficiently packed, highly dense B2-Form II and B1 crystals display $\beta$ values that are $<80 \mathrm{TPa}^{-1}$.

Overall, the computed complexation energies and bulk DFT estimates of the cocrystal stabilization energies suggest favorable thermodynamics for constructing higher-order cocrystals. The variations in the computed mechanical properties of these hybrid cocrystals reflect the differences in the intermolecular forces binding the chemical fragments together and suggest that careful choice of the coformers can be used to tune the properties of the cyclodiphosphazane cocrystal.

\section{Conclusions}

In summary, this study has shown how mechanochemistry can be used to rapidly screen, access and transform hybrid multicomponent cocrystals from individual components as well as from lower-order counterparts. We show that the solvent-less synthesis of ternary and quaternary hybrid organic-inorganic cocrystals can be achieved efficaciously in quantitative yield by both direct and telescopic routes by simply grinding the appropriate chemical species in a ball-mill. It is important to reiterate that such synthetic versatility of using binary or ternary cocrystals as reagents cannot be conducted using conventional solution methods, but is easily achieved using mechanochemistry. Moreover, we report the first example of the mechanochemical synthesis of a quaternary hybrid organic-inorganic cocrystal sustained by orthogonal ion-dipole, halogen- or hydrogenbonding interactions. All cocrystals were synthesized by utilizing the versatile Se-oxidized cyclodiphospha(V/V)zane molecules as building blocks, capitalizing on the formation of robust supramolecular synthons such as the bifurcated hydrogen bonds, halogen bonds, Se- $\pi$ and ion-dipole interactions. Furthermore, each building block in the complex quaternary cocrystal is structurally unique, having great potential for further expansion if each component's isostructurality is considered.

Computational modelling of the energetics of molecular association suggested that all higher-order cocrystals are driven to form on the basis of favorable thermodynamics. The calculated mechanical properties in the resulting cocrystals vary according to the chemical constituents of the solids, as well as the strength of the intermolecular forces present. These observations suggest the potential to optimize bulk material properties via the synthesis of higher-order cocrystals through the precise selection of the chemical constituents within the crystals.
Our work underscores the topological flexibility of phosphazane main group frameworks in enabling access to complex molecular solids. We hope that these results act as a platform for future development of more elaborate phosphazane building blocks towards the synthesis of more complex higher-order supramolecular arrangements and phosphazane-based hybrid organic-inorganic functional materials.

\section{ASSOCIATED CONTENT}

Experimental details, powder and single crystal X-Ray data (CCDC 194380o, 1967553, 2012787, and 20122788), diffraction patterns, spectroscopic data, and computational details are given in the Supporting Information (SI).

\section{AUTHOR INFORMATION}

† Both authors contributed equally

\section{Corresponding Author}

* sharmarke.mohamed@ku.ac.ae and fgarcia@ntu.edu.sg.

\section{ACKNOWLEDGMENT}

F.G. would like to thank A*STAR AME IRG (A1783c0oos and A2083c0050) and a NTU start-up grant (M4080552) for financial support. S.M. would like to acknowledge Khalifa University for financial support under the CIRA program (Project Code: CIRA-2018-o68). The theoretical calculations were performed using the high-performance computing clusters of Khalifa University and the authors would like to acknowledge the support of the research computing department.

\section{REFERENCES}

[1] Aitipamula, S.; Banerjee, R.; Bansal, A. K.; Biradha, K.; Cheney, M. L.; Choudhury, A. R.; Desiraju, G. R.; Dikundwar A. G.; Dubey, R.; Duggirala, N.; Ghogale, P. P.; Ghosh, S.; Goswami, P. K.; Goud, N. R.; Jetti, R. R. K. R.; Karpinski, P.; Kaushik, P.; Kumar, D.; Kumar, V.; Moulton, B.; Mukherjee, A.; Mukherjee, G.; Myerson, A. S.; Puri, V.; Ramanan, A.; Rajamannar, T.; Reddy, C. M.; Rodriguez-Hornedo, N.; Rogers, R. D.; Row, T. N. G.; Sanphui, P.; Shan, N.; Shete, G.; Singh, A.; Sun, C. C.; Swift, J. A.; Thaimattam, R.; Thakur, T. S.; Kumar Thaper, R.; Thomas, S. P.; Tothadi, S.; Vangala, V. R.; Variankaval, N.; Vishweshwar, P.; Weyna, D. R.; Zaworotko, M. J., Polymorphs, Salts, and Cocrystals: What's in a Name? Cryst. Growth Des., 2012, 12, 2147-2152.

[2] Clarke, H. D.; Hickey, M. B.; Moulton, B.; Perman, J. A.; Peterson, M. L.; Wojtas, Ł.; Almarsson, Ö.; Zaworotko, M. J., Crystal Engineering of Isostructural Quaternary Multicomponent Crystal Forms of Olanzapine. Cryst. Growth Des., 2012, 12, 41944201.

[3] Grothe, E.; Meekes, H.; Vlieg, E.; ter Horst, J. H.; de Gelder, R., Solvates, Salts, and Cocrystals: A Proposal for a Feasible Classification System. Cryst. Growth Des., 2016, 16, 32373243.

[4] Zhang, C.; Xiong, Y.; Jiao, F.; Wang, M.; Li, H., Redefining the Term of "Cocrystal" and Broadening Its Intention. Crystal Growth E Design, 2019, 19, 1471-1478.

[5] He, G.; Chow, P. S.; Tan, R. B. H., Predicting Multicomponent Crystal Formation: The Interplay between Homomeric and Heteromeric Interactions. Crystal Growth $\mathcal{E}$ Design, 2009, 9, 4529-4532. 
[6] Tothadi, S.; Mukherjee, A.; Desiraju, G. R., Shape and size mimicry in the design of ternary molecular solids: towards a robust strategy for crystal engineering. Chem. Commun., 2011, 47, 12080-12082.

[7] Bolla, G.; Nangia, A., Multicomponent ternary cocrystals of the sulfonamide group with pyridine-amides and lactams. Chem. Commun., 2015, 51, 15578-15581.

[8] Dubey, R.; Mir, N. A.; Desiraju, G. R., Quaternary cocrystals: combinatorial synthetic strategies based on long-range synthon Aufbau modules (LSAM). IUCrJ, 2016, 3, 102-107

[9] Goswami, P. K.; Thaimattam, R.; Ramanan, A., Crystal Engineering of Multicomponent Crystal Forms of pAminosalicylic Acid with Pyridine Based Coformers. Cryst. Growth Des., 2016, 16, 1268-1281.

[10] Berry, D. J.; Steed, J. W., Pharmaceutical cocrystals, salts and multicomponent systems; intermolecular interactions and property based design. Adv. Drug Deliv. Rev., 2017, 117, 3-24.

[11] N. Schultheiss, A. Newman, Pharmaceutical Cocrystals and Their Physicochemical Properties. Cryst. Growth Des., 2oo9, 9, $2950-2967$.

[12] Friscic, T.; Jones, W., Benefits of cocrystallization in pharmaceutical materials science: an update. J. Pharm. Pharmacol., 2010, 62, 1547-1559.

[13] Yan, D.; Delori, A.; Lloyd, G. O.; Friscic, T.; Day, G. M.; Jones, W.; Lu, J.; Wei, M.; Evans, D. G.; Duan, X., A cocrystal strategy to tune the luminescent properties of stilbene-type organic solid-state materials. Angew. Chem. Int. Ed., 2011, 50 , 12483-12486.

[14] Bolton, O.; Matzger, A. J., Improved stability and smartmaterial functionality realized in an energetic cocrystal. Angew. Chem. Int. Ed., 2011, 50, 8960-8963.

[15] Tayi, A. S.; Shveyd, A. K.; Sue, A. C.; Szarko, J. M.; Rolczynski, B. S.; Cao, D.; Kennedy, T. J.; Sarjeant, A. A.; Stern, C. L.; Paxton, W. F.; Wu, W.; Dey, S. K.; Fahrenbach, A. C.; Guest, J. R.; Mohseni, H.; Chen, L. X.; Wang, K. L.; Stoddart, J. F.; Stupp, S. I., Room-temperature ferroelectricity in supramolecular networks of charge-transfer complexes. Nature, 2012, 488, 485-489.

[16] Bushuyev, O. S.; Corkery, T. C.; Barrett, C. J.; Friščić, T., Photo-mechanical azobenzene cocrystals and in situ X-ray diffraction monitoring of their optically-induced crystal-tocrystal isomerization. Chem. Sci., 2014, 5, 3158-3164.

[17] Aakeröy, C. B.; Beatty, A. M.; Helfrich, B. A., "Total Synthesis" Supramolecular Style: Design and Hydrogen-BondDirected Assembly of Ternary Supermolecules. Angewandte Chemie International Edition 2001, 40 (17), 3240-3242.

[18] Mandal, S.; Mukhopadhyay, T. K.; Mandal, T.; A. Datta. Hierarchical Noncovalent Interactions between Molecules Stabilize Multicomponent Cocrystals. Cryst. Growth Des., 2019, 19, 4802-4809.

[19] Bolla, G; Nangia, A., Multicomponent ternary cocrystals of the sulfonamide group with pyridine-amides and lactams, Chem. Commun., 2015, 51, 15578-15581.

[20] Mir, N. A.; Dubey, R.; Desiraju, G. R., Four- and fivecomponent molecular solids: crystal engineering strategies based on structural inequivalence. IUCrJ, 2016, 3, 96-101.

[21] Paul, M.; Chakraborty, S.; Desiraju, G. R., Six-

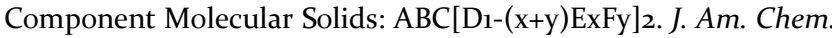
Soc., 2018, 140, 2309-2315.

[22] Mir, N. A.; Dubey, R.; Desiraju, G. R., Strategy and Methodology in the Synthesis of Multicomponent Molecular Solids: The Quest for Higher Cocrystals. Acc. Chem. Res., 2019, 52, $8,2210-2220$.

[23] Paul, M; Desiraju, G. R., From a Binary to a Quaternary Cocrystal: An Unusual Supramolecular Synthon, Angew. Chem. Int. Ed., 2019, 58, 12027-12031.
[24] d'Agostino, S.; Fornasari, L.; Braga, D., Binary and Ternary Solid Solutions of Ionic Plastic Crystals, and Modulation of Plastic Phase Transitions Cryst. Growth Des., 2019, 19, 62666273 .

[25] Lusi, M., A rough guide to molecular solid solutions: design, synthesis and characterization of mixed crystals CrystEngComm, 2018, 20, 7042-7052.

[26] Lusi, M., Engineering Crystal Properties through Solid Solutions. Cryst. Growth Des., 2018, 18, 3704-3712.

[27] Winscons, R. A.; Coropceanu, V.; Matzger, A. J., Quaternary Charge-Transfer Solid Solutions: Electronic Tunability through Stoichiometry, Chem. Mater., 2019, 31, 65986604.

[28] Topić, F.; Rissanen, K., Systematic Construction of Ternary Cocrystals by Orthogonal and Robust Hydrogen and Halogen Bonds. J. Am. Chem. Soc., 2016, 138, 6610-6616.

[29] Braga, D.; Grepioni, F.; Shemchuk, O., Organicinorganic ionic cocrystals: a new class of multipurpose compounds. CrystEngComm, 2018, 20, 2212-2220.

[30] Wang, T.; Stevens, J. S. S.; Vetter, T.; Whitehead, G. F. S; Vitorica-Yrezabal, I. J.; Hao, H.; Cruz-Cabeza, A. J., Salts, Cocrystals, and Ionic Cocrystals of a "Simple" Tautomeric Compound. Cryst. Growth Des., 2018, 18, 6973-6983.

[31] Lixing Song, L.; Robeyns, K.; Leyssens, T., Crystallizing Ionic Cocrystals: Structural Characteristics, Thermal Behavior, and Crystallization Development of a Piracetam- $\mathrm{CaCl}_{2}$ Cocrystallization Process, Cryst. Growth Des. 2018, 18, 3215-3221.

[32] Balakrishna, M. S., Cyclodiphosphazanes: options are endless. Dalton Trans., 2016, 45, 12252-12282.

[33] Shi, X.; León, F.; Sim, Y.; Quek, S.; Hum, G.; Khoo, Y. X J.; Ng, Z. X.; Par, M. Y.; Ong, H. C.; Singh, V. K.; Ganguly R.; Clegg, J. K.; Díaz, J.; García, F. N-Bridged Acyclic Trimeric PolyCyclodiphosphazanes: Highly Tuneable Cyclodiphosphazane Building Blocks. Angew. Chem, Int. Ed., 2020, 59, 22100-22108.

[34] Tan, D.; Ng, Z. X.; Sim, Y.; Ganguly, R.; García, F., cisCyclodiphosph(v/v)azanes as highly stable and robust main group supramolecular building blocks. CrystEngComm., 2018, 20, 59986004 .

[35] Friscic, T., Supramolecular concepts and new techniques in mechanochemistry: cocrystals, cages, rotaxanes, open metal-organic frameworks. Chem. Soc. Rev., 2012, 41, 34933510.

[36] Delori, A.; Friščić, T.; Jones, W., The role of mechanochemistry and supramolecular design in the development of pharmaceutical materials. CrystEngComm, 2012, 14, 2350-2362.

[37] Ayoub, G.; Karadeniz, B.; Howarth, A. J.; Farha, O. K.; Đilović, I.; Germann, L. S.; Dinnebier, R. E.; Užarević, K.; Friščić, T., Rational Synthesis of Mixed-Metal Microporous MetalOrganic Frameworks with Controlled Composition Using Mechanochemistry, Chem. Mater., 2019, 31,5494-5501.

[38] Julien, P. A.; Mottillo, C.; Friščić, T., Metal-organic frameworks meet scalable and sustainable synthesis, Green Chem., 2017, 19, $2729-2747$.

[39] Rath, B. B.; Kole, G. K.; Morris, S. A.; Vittal, J. J., Rotation of a helical coordination polymer by mechanical grinding, Chem. Commun., 2020, 56, 6289-6292.

[40] Friščić, T.; Trask, A. V.; Jones, W.; Motherwell, W. D., Screening for inclusion compounds and systematic construction of three-component solids by liquid-assisted grinding. Angew. Chem. Int. Ed., 2006, 45, 7546-7550.

[41] Trask, A. V.; Haynes, D. A.; Motherwell, W. D.; Jones, W., Screening for crystalline salts via mechanochemistry. Chem. Commun. 2006, 51-53. 
[42] Hasa, D.; Rauber, G. S.; Voinovich, D.; Jones, W., Cocrystal Formation through Mechanochemistry: from Neat and Liquid-Assisted Grinding to Polymer-Assisted Grinding. Angew. Chem. Int. Ed., 2015, 54, 7371-5.

[43] Hasa, D.; Jones, W., Screening for new pharmaceutical solid forms using mechanochemistry: A practical guide. Adv. Drug. Deliv. Rev., 2017, 117, 147-161.

[44] Friščić, T.; Lancaster, R. W.; Fabian, L.; Karamertzanis, P. G., Tunable recognition of the steroid alpha-face by adjacent pi-electron density. Proc. Natl. Acad. Sci. U.S.A., 2010, 107, 1321613221.

[45] Cheung, E. Y.; S. J. K., Harris, K. D. M.; Imai, Y.; Tajima, N.; Kuroda, R.. Direct Structure Determination of a Multicomponent Molecular Crystal Prepared by a Solid-State Grinding Procedure. J. Am. Chem. Soc., 2oo3, 125, 14658-14659.

[46] Cinčić, D.; Friščić, T.; Jones, W. A Stepwise Mechanism for the Mechanochemical Synthesis of Halogen-Bonded Cocrystal Architectures. J. Am. Chem. Soc., 2008, 130, 7524-7525.

[47] Weyna, D. R.; Shattock, T.; Vishweshwar, P.; Zaworotko, M. J. Synthesis and Structural Characterization of Cocrystals and Pharmaceutical Cocrystals: Mechanochemistry vs Slow Evaporation from Solution. Cryst. Growth Des., 2009, 9, 1106-1123.

[48] Friščić, T., New opportunities for materials synthesis using mechanochemistry. J. Mat. Chem., 2010, 20, 7599-7605.

[49] Braga, D.; Maini, L.; Grepioni, F., Mechanochemical preparation of cocrystals. Chem. Soc. Rev., 2013, 42, 7638-7648.

[50] Hayashi, Y., Pot economy and one-pot synthesis, Chem. Sci., 2016,7, 866-88o.

[51] Shi, Y.-X.; Martin, K. A.; Weston, N..; Gonzalez-Calera, S.; Ganguly, R.; Li, Y.; Lu, Y.; Ribeiro, A.; Ramos, M.; Fernandes, P.; García, F. Synthesis and hydrolytic studies on the air-stable [(4$\left.\mathrm{CN}-\mathrm{PhO}) \mathrm{P}(\mathrm{E})\left(\mu-\mathrm{N}^{t} \mathrm{Bu}\right)\right]_{2}(\mathrm{E}=\mathrm{O}, \mathrm{S}$ and $\mathrm{Se})$ cyclodiphosphazanes. Inorg. Chem., 2015, 54, 6423-6432.

[52] Sim, Y.; Shi, Y.-X.; Ganguly, R.; Li, Y.; García, F. Mechanochemical Synthesis of Phosphazane-based Compounds. Chem. Eur. J., 2017, 47, 11279-11285.

[53] Sim, Y.; Tan, D.; Ganguly, R.; Li, Y.; García, F. Orthogonality in Main Group Compounds: Direct One-step Synthesis of Air- and Moisture-stable Cyclodiphosphazane s by Mechanochemistry. Chem. Commun., 2018, 20, 5998-6004.

[54] Klare, H.; Hanft, S.; Neudörfl, J. M.; Schlörer, N. E.; Griesbeck, A.; nd Goldfuss, B. Anion Recognition with HydrogenBonding Cyclodiphosphazanes. Chem. Eur. J., 2014, 20, 1184711855 .

[55] Plajer, A. J.; Zhu, J.; Proehm, P.; Bond, A. D.; Keyser, U. F.; Wright, D. S. Tailoring the Binding Properties of Phosphazane Anion Receptors and Transporters. J. Am. Chem. Soc. 2019, 141, 8807-8815.

[56] Plajer, A. J.; Zhu, J.; Proehm, P.; Bond, A. D.; Keyser, U. F.; Wright, D. S. Syntheses and X-ray Structures of Potassium Derivatives and a Paramagnetic Nickel(II) Complex of a Cyclodiphosph(III/V)azane Monoselenide Inorg. Chem., 2002, 41, 4348-4354.

[57] Throughout the text, $\mathbf{S e}_{2} \mathbf{P}_{2} \mathbf{N}_{\mathbf{2}}$ corresponds to $\mathbf{S e}_{2} \mathbf{P}_{2} \mathbf{N}_{\mathbf{2}}$ exo, exo unless otherwise stated.

[58] Aakeroy, C. B.; Baldrighi, M.; Desper, J.; Metrangolo, P.; Resnati, G., Supramolecular hierarchy among halogen-bond donors. Chem. Eur. J., 2013, 19, 16240-16247.

[59] Bucar, D. K.; Sen, A.; Mariappan, S. V.; MacGillivray, L. R., A [2+2] cross-photodimerization of photostable olefins via a three-component cocrystal solid solution. Chem. Commun., 2012, 48, 1790-1792.

[6o] L. Loots, H. W., L. Westhuizen, D. A. Haynes, T. L. Roex, Interconversion between different stoichiometric forms of a three-component crystal via liquid-assisted grinding. Chem Commun., 2012, 48, 11507-11509.

[61] Smith, A. J.; Kim, S. H.; Duggirala, N. K.; Jin, J.; Wojtas, L.; Ehrhart, J.; Giunta, B.; Tan, J.; Zaworotko, M. J.; Shytle, R. D., Improving lithium therapeutics by crystal engineering of novel ionic cocrystals. Mol. Pharmaceutics, 2013, 10, 4728-38.

[62] Buist, A. R.; Kennedy, A. R., Ionic Cocrystals of Pharmaceutical Compounds: Sodium Complexes of Carbamazepine. Cryst. Growth Des., 2014, 14, 6508-6513.

[63] Song, L.; Robeyns, K.; Leyssens, T., Crystallizing Ionic Cocrystals: Structural Characteristics, Thermal Behavior, and Crystallization Development of a Piracetam- $\mathrm{CaCl}_{2}$ Cocrystallization Process. Cryst. Growth Des., 2018, 18, 3215-3221.

[64] Bukenya, S.; Munshi, T.; Scowen, I. J.; Skyner, R.; Whitaker, D. A.; Seaton, C. C., Building multicomponent crystals from cations and cocrystals: the use of chaperones. CrystEngComm, 2013, 15, 2241-2250.

[65] Duer, M. J.; García, F.; Kowenicki, R. A.; Naseri, V.; McPartlin, M.; Stead, M. L.; Stein, R. S.; Wright, D. S., Inverse coordination of an ionic lattice by a metal host. Angew. Chem. Int. Ed., 2005, 44, 5729-5733.

[66] García, F.; Less, R. J.; McPartlin, M.; Michalski, A.; Mulvey, R. E.; Naseri, V.; Stead, M. L.; Moran de Vega, A.; Wright, D. S., Confinement of halide ions within homologous inverse coordination hosts; modification of halide-ion selectivity. Chem. Commun., 2011, 47, 1821-3.

[67] T. Chivers, T.; Krahn, M.; Parvez, M.; Schatte, G. Preparation and X-ray Structures of Alkali-Metal Derivatives of the Ambidentate Anions [ $\left.{ }^{\mathrm{t}} \mathrm{BuN}(\mathrm{E}) \mathrm{P}\left(\mu-\mathrm{N}^{\mathrm{t}} \mathrm{Bu}\right)_{2} \mathrm{P}(\mathrm{E}) \mathrm{N}^{\mathrm{t} B u}\right]^{2-}(\mathrm{E}=\mathrm{S}$, $\mathrm{Se})$ and $\left.\left[{ }^{\mathrm{t}} \mathrm{BuN}(\mathrm{Se}) \mathrm{P}\left(\mu-\mathrm{N}^{\mathrm{t}} \mathrm{Bu}\right)_{2} \mathrm{PN}(\mathrm{H})^{\mathrm{t}} \mathrm{Bu}\right)\right]^{-}$. Inorg. Chem., 20o1, 40 , 2547-2553.

[68] T. Chivers, T.; Krahn, Schatte, G. Syntheses and X-ray Structures of Potassium Derivatives and a Paramagnetic Nickel(II) Complex of a Cyclodiphosph(III/V)azane Monoselenide. Inorg. Chem., 20o2, 41, 4348-4354.

[69] Klare, H.; Hanft, S.; Neudorfl, J. M.; Schlorer, N. E.; Griesbeck, A.; Goldfuss, B., Anion recognition with hydrogenbonding cyclodiphosphazanes. Chem. Eu. J., 2014, 20, 11847-55.

[7o] Childs, S. L.; Chyal, L. J.; Dunlap, J. T.; Smolenskaya, V. N.; Stahly, B. C.; G. P. Crystal Engineering Approach To Forming Cocrystals of Amine Hydrochlorides with Organic Acids. Molecular Complexes of Fluoxetine Hydrochloride with Benzoic, Succinic, and Fumaric Acids. J. Am. Chem. Soc., 2004, 126, 1333513342.

[71] Braga, D.; Grepioni, F.; Maini, L.; Prosperi, S.; Gobetto, R.; Chierotti, M. R., From unexpected reactions to a new family of ionic cocrystals: the case of barbituric acid with alkali bromides and caesium iodide. Chem. Commun., 2010, 46, 7715-7.

[72] Braga, D.; Grepioni, F.; Lampronti, G. I.; Maini, L.; Turrina, A., Ionic Co-crystals of Organic Molecules with Metal Halides: A New Prospect in the Solid Formulation of Active Pharmaceutical Ingredients. Cryst. Growth Des., 2011, 11, 56215627 .

[73] Ong, T. T.; Kavuru, P.; Nguyen, T.; Cantwell, R.; Wojtas, L.; Zaworotko, M. J., 2:1 cocrystals of homochiral and achiral amino acid zwitterions with $\mathrm{Li}+$ salts: water-stable zeolitic and diamondoid metal-organic materials. J. Am. Chem. Soc., 2011, 133, 9224-7.

[74] Aoki, S.; Sasaki, S.; Koga, K. Simple chiral crown ethers complexed with potassium tert-butoxide as efficient catalysts for asymmetric Michael additions. Tetrahedron Lett. 1989, 30, 7229.

[75] Cram, D. J. The design of molecular hosts, guests, and their complexes. Science, 1998, 240, 760-767. 
[76] Gokel, G. W.; Leevy, W. M.; Weber, M. E. Crown Ethers: Sensors for Ions and Molecular Scaffolds for Materials and Biological Models. Chem. Rev., 2004, 104, 2723-2750.

[77] Ward, M. D. Design of crystalline molecular networks with charge-assisted hydrogen bonds. Chem. Commun., 2005, 5838-42.

[78] Aakeröy, C. B.; Fasulo, M.; Schultheiss, N.; Desper, J.; Moore, C. Structural Competition between Hydrogen Bonds and Halogen Bonds. J. Am. Chem. Soc., 2007, 129, 13772-13773.

[79] Aakeroy, C. B.; Spartz, C. L.; Dembowski, S.; Dwyre, S.; Desper, J., A systematic structural study of halogen bonding versus hydrogen bonding within competitive supramolecular systems. IUCrJ, 2015, 2, 498-510.

[8o] Cavallo, G.; Metrangolo, P.; Milani, R.; Pilati, T.; Priimagi, A.; Resnati, G.; Terraneo, G., The Halogen Bond. Chem. Rev., 2016, 116, 2478-601.

[81] Hernández, J. G.; Butler, I. S.; Friščić, T., Multi-step and multicomponent organometallic synthesis in one pot using orthogonal mechanochemical reactions, Chem. Sci., 2o14, 5, 35763582 .
[82] Taylor, C. R.; Day, G. M., Evaluating the Energetic Driving Force for Cocrystal Formation. Cryst. Growth Des., 2018, 18, 892904.

[83] Mohamed, S.; Alwan, A. A.; Friščić, T.; Morris, A. J.; Arhangelskis, M., Towards the systematic crystallization of molecular ionic cocrystals: insights from computed crystal form landscapes. Faraday Discuss., 2018, 211, 401-424.

[84] Shunnar, A. F.; Dhokale, B.; Karothu, D. P.; Bowskill, D. H.; Sugden, I. J.; Hernandez, H. H.; Naumov, P.; Mohamed, S., Efficient Screening for Ternary Molecular Ionic Cocrystals Using a Complementary Mechanosynthesis and Computational Structure Prediction Approach. Chem. Eu. J., 2o2o, 26, 4752-4765.

[85] Sun, C.; Grant, D. J. W., Improved Tableting Properties of p-Hydroxybenzoic Acid by Water of Crystallization: A Molecular Insight. Pharm. Res., 2004, 21, 382-386. 
SYNOPSIS TOC (Word Style "SN_Synopsis_TOC"). If you are submitting your paper to a journal that requires a synopsis graphic and/or synopsis paragraph, see the Instructions for Authors on the journal's homepage for a description of what needs to be provided and for the size requirements of the artwork.

To format double-column figures, schemes, charts, and tables, use the following instructions:

Place the insertion point where you want to change the number of columns

From the Insert menu, choose Break

Under Sections, choose Continuous

Make sure the insertion point is in the new section. From the Format menu, choose Columns

In the Number of Columns box, type 1

Choose the OK button

Now your page is set up so that figures, schemes, charts, and tables can span two columns. These must appear at the top of the page. Be sure to add another section break after the table and change it back to two columns with a spacing of 0.33 in.

Table 1. Example of a Double-Column Table

\begin{tabular}{|l|l|l|l|l|l|l|l|}
\hline Column 1 & Column 2 & Column 3 & Column 4 & Column 5 & Column 6 & Column 7 & Column 8 \\
\hline & & & & & & & \\
\hline
\end{tabular}

Authors are required to submit a graphic entry for the Table of Contents (TOC) that, in conjunction with the manuscript title, should give the reader a representative idea of one of the following: A key structure, reaction, equation, concept, or theorem, etc., that is discussed in the manuscript. Consult the journal's Instructions for Authors for TOC graphic specifications.

\section{Insert Table of Contents artwork here}

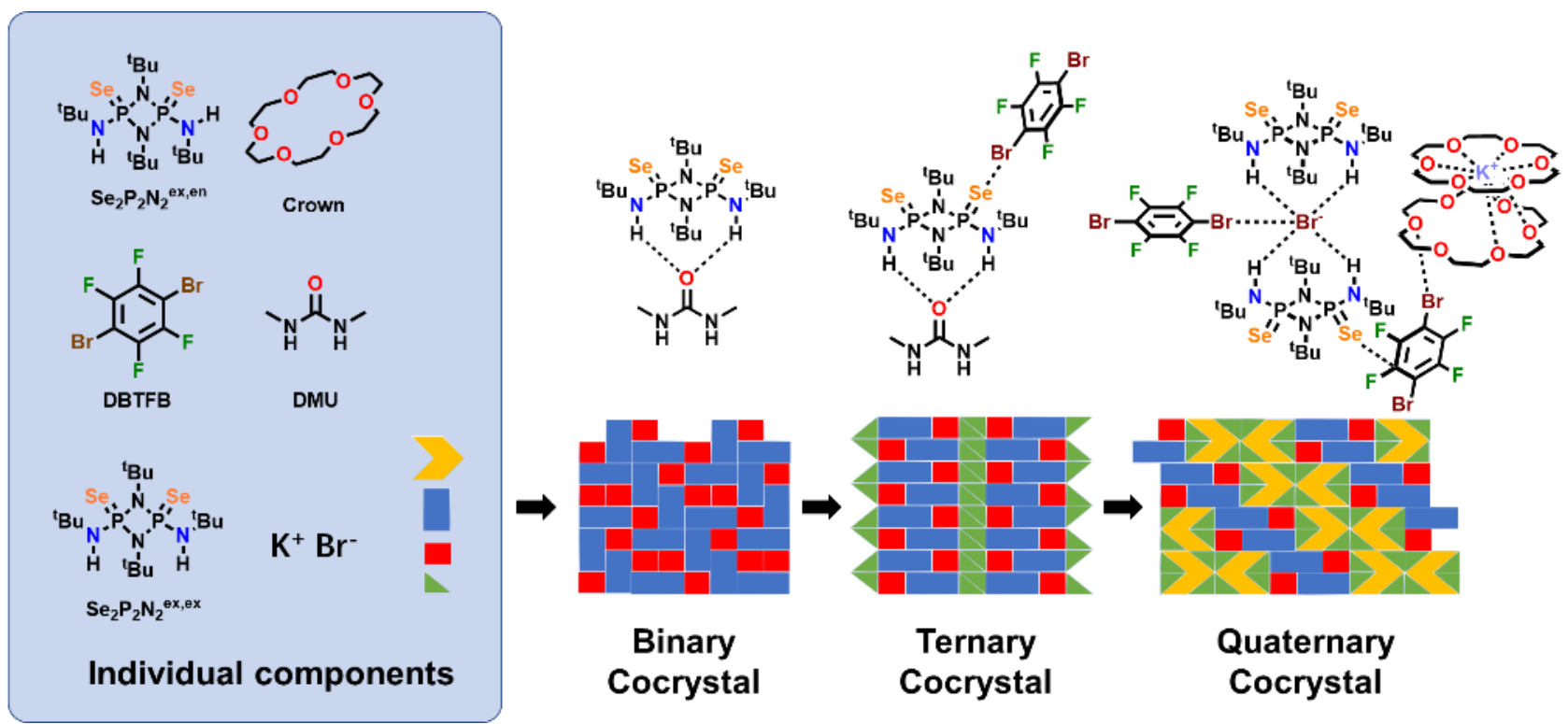

